\title{
Hypercyclicity of Semigroups is a Very Unstable Property
}

\author{
W. Desch ${ }^{1}$ and W. Schappacher \\ Karl-Franzens-Universität Graz, Institut für Mathematik und wissenschaftliches Rechnen \\ Heinrichstraße 36, A-8010 Graz, Austria
}

\begin{abstract}
Hypercyclicity of $C_{0}$-semigroups is a very unstable property: We give examples to show that adding arbitrary small constants or a bounded rank one operator to the generator of a hypercyclic semigroup can destroy hypercyclicity. Also the limit of hypercyclic semigroups (even in operator norm topology) need not be hypercyclic, and a hypercyclic semigroup can be the limit of nonhypercyclic ones. Hypercyclicity is not inherited by the Yosida approximations. Finally, the restriction of a hypercyclic nonnegative semigroup in a Banach lattice to the positive cone may be far from hypercyclic.
\end{abstract}

Key words: hypercyclic semigroups, perturbation

AMS subject classification: 47A16, 47D03

\section{Introduction and main results}

Throughout this paper, let $X$ be a real or complex (infinite dimensional) Banach space. A family $S(t)$ of continuous linear operators on $\mathrm{X}$ is called a strongly continuous semigroup iff it satisfies

$$
\begin{aligned}
& S(0)=\mathrm{id}, \\
& S(t+s)=S(t) S(s) \text { for all nonnegative s,t, } \\
& S(t) x \text { depends continuously on } t \text { for all } x .
\end{aligned}
$$

For a concise treatment of strongly continuous semigroups we refer to [7]. In the last years we see an increasing interest in the analysis and applications of irregularly behaved solutions. An

\footnotetext{
${ }^{1}$ Corresponding author. E-mail: georg.desch@uni-graz.at
} 
extreme case of instability and irregular behavior is observed in the case of hypercyclic semigroups. Just to mention a few recent references on this topic and its applications we cite [1], [2], [3], [6], [8], [9].

Definition 1. The semigroup $S(t)$ is called hypercyclic iff there exists some vector $x$ such that its orbit $\{S(t) x \mid t \geq 0\}$ is dense in $X$. Any such vector $x$ is said to be a hypercyclic vector for $S(t)$.

This paper is a collection of examples which show that hypercyclicity is a very fragile property, which is destroyed by very small perturbations.

Our first example shows that hypercyclicity may be destroyed even by bounded perturbations of rank one:

Example 2. For any hypercyclic semigroup $S(t)$ with infinitesimal generator $A$ there exists a bounded linear operator $B$ such that the semigroup generated by $A+B$ is not hypercyclic.

The (surprisingly simple) details of this result as well as the details of the following examples will be given in Section 2 ..

It is clear that for any hypercyclic semigroup $S(t)$ generated by $A$ the perturbation $A+\lambda$ id may generate a non-hypercyclic semigroup. For instance, take $\lambda$ sufficiently negative, so that $e^{\lambda t} S(t)$ is exponentially stable. However, it is surprising that there may be an interval such that $e^{\lambda t} S(t)$ is hypercyclic for all $\lambda$ in the whole interval with the exception of isolated points:

Example 3. There exists a semigroup $S(t)$ which is not hypercyclic, such that for all sufficiently small $\lambda>0$ the semigroups $e^{\lambda t} S(t)$ and $e^{-\lambda t} S(t)$ are hypercyclic.

Moreover, limits of hypercyclic semigroups need not be hypercyclic, and limits of non-hypercyclic semigroups may be hypercyclic. Thus, in the sense of Kato-Trotter convergence, neither the hypercyclic semigroups nor the non-hypercyclic semigroups form an open set.

Example 4. There exists a semigroup $S(t)$ which is not hypercyclic, and a sequence of hypercyclic semigroups $\left(S_{n}(t)\right)$, such that $\left\|S_{n}(t)-S(t)\right\|$ converges to 0 uniformly for $t$ in compact intervals.

In fact, even the Yosida approximation of a hypercyclic semigroup need not inherit hypercyclicity: 
Example 5. There exists a a hypercyclic semigroup $S(t)$ with infinitesimal generator $A$ with the following properties: For all $\lambda>1$, the Yosida approximations $A_{\lambda}=\lambda A(\lambda-A)^{-1}$ generate semigroups which are not hypercyclic.

In particular, a hypercyclic semigroup may be the limit of non-hypercyclic semigroups (in the Kato-Trotter sense).

In order to motivate the next property, let us consider a situation which occurs frequently in applications. The semigroup under investigation describes the time evolution of a physical or biological process which is characterized by a densitiy function. Typically, hypercyclicity can be shown by spectral methods in a complex space. However, physical or biological interest centers only on nonnegative solutions. Thus, consider a (real) Banach lattice $X$ with a nonnegative semigroup $S(t)$, and let $\tilde{X}$ be its complexification. The extension of $S(t)$ to $\tilde{X}$ is called $\tilde{S}(t)$. It is easily seen that hypercyclicity of $\tilde{S}$ on $\tilde{X}$ implies that $S$ is hypercyclic on $X$. However, this does not imply hypercyclicity of the restriction of $S$ to the positive cone:

Example 6. There exists a Banach lattice $X$ with positive cone $X^{+}$and a hypercyclic semigroup $S(t)$ which is nonnegative in the sense that $S(t)\left(X^{+}\right) \subset X^{+}$, however such that the trajectories contained in the positive cone are nondecreasing. In particular, $X^{+}$does not contain a dense trajectory.

\section{The examples in detail}

\subsection{Example 2}

Recall that a semigroup $T(t)$ cannot be hypercyclic if the point spectrum of $A^{*}$ is nonempty ([5]). So, let $A$ be the infinitesimal generator of a hypercyclic semigroup on a Banach space $X$. Let $\langle\cdot, \cdot\rangle$ denote the pairing between the dual space $X^{*}$ and $X$. We choose some $v \in X$ and $u^{*} \in D\left(A^{*}\right)$ such that $\left\langle u^{*}, v\right\rangle=-1$. We define a bounded rank one operator $B$ by

$$
B x:=A x+\left\langle A^{*} u^{*}, x\right\rangle v .
$$

Then $A+B$ (defined on $D(A)$ ) is again the infinitesimal generator of an analytic semigroup on $X$. However, since for all $x \in D(A)$

$$
\left\langle u^{*},(A+B) x\right\rangle=\left\langle u^{*}, A x\right\rangle+\left\langle A^{*} u^{*}, x\right\rangle\left\langle u^{*}, v\right\rangle=0
$$

we infer that $u^{*}$ is an eigenvector of $(A+B)^{*}$ with eigenvalue 0 . Therefore $B$ cannot be the generator of a hypercyclic semigroup on $X$. 


\subsection{Translation semigroups}

Translation semigroups are a good source of examples, since for these semigroups there exists a particularly easy characterization for hypercyclicity.

Definition 7. For a function $f: \mathbb{R} \rightarrow \mathbb{R}$ or $f:[0, \infty) \rightarrow \mathbb{R}$ and $t \geq 0$ we define the left translation $T(t) f$ by

$$
[T(t) f](\tau)=f(t+\tau) \quad \text { for } \tau \in \mathbb{R} \text { or } \tau \in[0, \infty), \text { respectively. }
$$

We introduce suitable Banach spaces such that this left translation forms a semigroup:

Definition 8. A measurable function $\rho: \mathbb{R} \rightarrow(0, \infty)$ is called an admissible weight function on $\mathbb{R}$ iff there exist some $M \geq 1$ and $\omega \geq 0$ such that for all $t \in \mathbb{R}$ and $h \geq 0$ we have

$$
\rho(t+h) \geq M e^{-\omega h} \rho(t) \quad \text { and } \quad \rho(t-h) \geq M e^{-\omega h} \rho(t) .
$$

A measurable function $\rho:[0, \infty) \rightarrow(0, \infty)$ is called an admissible weight function on $[0, \infty)$ iff there exist some $M \geq 1$ and $\omega \geq 0$ such that for all $t \geq 0$ and $h \geq 0$ we have

$$
\rho(t+h) \geq M e^{-\omega h} \rho(t) .
$$

Definition 9. Let $\rho$ be an admissible weight function on $\mathbb{R}$. For $p \in[1, \infty)$, we define

$$
\begin{aligned}
L_{\rho}^{p}(\mathbb{R}) & =\left\{f: \mathbb{R} \rightarrow \mathbb{R} \mid \text { f measurable and } \int_{\mathbb{R}} \rho(t)|f(t)|^{p} d t<\infty\right\}, \\
\|f\|_{p, \rho}^{p} & =\int_{\mathbb{R}} \rho(t)|f(t)|^{p} d t .
\end{aligned}
$$

An analogous definition is given for $L_{\rho}^{p}([0, \infty))$.

Definition 10. Let $\rho$ be an admissible weight function on $\mathbb{R}$. We define

$$
\begin{aligned}
\mathcal{C}_{0, \rho}(\mathbb{R}) & =\left\{f \in \mathcal{C}(\mathbb{R}, \mathbb{R})\left|\lim _{|t| \rightarrow \infty, t \in \mathbb{R}} \rho(t)\right| f(t) \mid=0\right\} \\
\|f\|_{\infty, \rho} & =\sup _{t \in \mathbb{R}} \rho(t)|f(t)|
\end{aligned}
$$

An analogous definition is given for $\mathcal{C}_{0, \rho}([0, \infty))$.

On these spaces, hypercyclicity of the left translation admits an easy characterization: 


\section{Theorem 11 ([5]).}

Let $\rho$ be an admissible weight function on $\mathbb{R}$, and let $X$ be one of the spaces $\mathcal{C}_{0, \rho}(\mathbb{R})$ or $L_{\rho}^{p}(\mathbb{R})$. The family $T(t)$ of left translations is a strongly continuous semigroup on X. Moreover, $T(t)$ is hypercyclic iff there exists a sequence $t_{n} \rightarrow \infty$ such that $\rho\left(t_{n}\right) \rightarrow 0$ and $\rho\left(-t_{n}\right) \rightarrow 0$.

Let $\rho$ be an admissible weight function on $[0, \infty)$, and let $X$ be one of the spaces $\mathcal{C}_{0, \rho}([0, \infty))$ or $L_{\rho}^{p}([0, \infty))$. The family $T(t)$ of left translations is a strongly continuous semigroup on $X$. Moreover, $T(t)$ is hypercyclic iff there exists a sequence $t_{n} \rightarrow \infty$ such that $\rho\left(t_{n}\right) \rightarrow 0$.

\subsection{Example 3}

We shall construct an admissible weight function $\rho$ such that the left translation $T(t)$ is not hypercyclic on $X=L_{\rho}^{p}(\mathbb{R})$, but for all sufficiently small $\lambda>0$, the semigroups $e^{\lambda t} T(t)$ and $e^{-\lambda t} T(t)$ are hypercyclic.

We first choose some $\mu \in \mathbb{R}$ and set $\sigma(t):=\exp (\mu \mathrm{t}) \rho(t), Y:=L_{\sigma}^{p}$ and $S(t)$ the left translation semigroup on $Y$.

Then the operator

$$
F: \begin{cases}X & \rightarrow Y \\ {[F(f)(s)]} & :=e^{-\frac{\mu s}{p}} f(s)\end{cases}
$$

is an isometric isomorphism and we have

$$
F^{-1} S(t) F=e^{-\frac{\mu t}{p}} T(t) .
$$

As a consequence, if we put $\mu:=\lambda p$, we see that $S(t)$ and hence also $e^{-\lambda t} T(t)$ are hypercyclic on $Y$ and $X$, respectively, iff there exists a sequence $t_{n} \rightarrow \infty$ such that both, $\sigma\left(t_{n}\right)=e^{p \lambda t_{n}} \rho\left(t_{n}\right)$ and $\sigma\left(-t_{n}\right)=e^{-p \lambda t_{n}} \rho\left(-t_{n}\right)$ converge to 0 as $n \rightarrow \infty$.

After this preparation, we are ready to construct the desired weight function $\rho(t)$ :

For $n=1,2,3, \cdots$ we put $s_{n}=2 \cdot 9^{n}$ and $t_{n}=6 \cdot 9^{n}$. This implies in particular that

$$
1=\frac{s_{1}}{2}<3 \frac{s_{1}}{2}=\frac{t_{1}}{2}<3 \frac{t_{1}}{2}=\frac{s_{2}}{2}<3 \frac{s_{2}}{2}=\frac{t_{2}}{2}<3 \frac{t_{2}}{2}=\frac{s_{3}}{2}<\cdots
$$

Now we set

$$
\rho(t)= \begin{cases}e^{-\frac{t_{i}}{2}+\left|t-t_{i}\right|} & \text { for } t \in\left[\frac{t_{i}}{2}, 3 \frac{t_{i}}{2}\right] \\ 1 & \text { for } t \in\left[\frac{s_{i}}{2}, 3 \frac{s_{i}}{2}\right] \\ 1 & \text { for } t \in[-1,1] \\ e^{-\frac{s_{i}}{2}+\left|t+s_{i}\right|} & \text { for } t \in\left[-3 \frac{s_{i}}{2},-\frac{s_{i}}{2}\right], \\ 1 & \text { for } t \in\left[-3 \frac{t_{i}}{2},-\frac{t_{i}}{2}\right] .\end{cases}
$$


Note that $\rho$ is continuous and admissible. The key idea is that whenever $\rho(-t)<1$ then we have $\rho(+t)=1$, and hence there is no sequence $\left(u_{n}\right)$ such that $\rho\left(u_{n}\right)$ and $\rho\left(-u_{n}\right)$ both converge to 0 . As a consequence, $T(t)$ is not hypercyclic on $X=L_{\rho}^{p}(\mathbb{R})$.

Now, choose any $\lambda \in\left(0, \frac{1}{2 p}\right)$. Then

$$
\begin{aligned}
& e^{p t_{i} \lambda} \rho\left(t_{i}\right)=e^{\left(p \lambda-\frac{1}{2}\right) t_{i}} \\
& e^{-p t_{i} \lambda} \rho\left(-t_{i}\right)=e^{-p t_{i} \lambda}
\end{aligned}
$$

Both sequences converge to 0 as $i \rightarrow \infty$, therefore $e^{\lambda t} T(t)$ is hypercyclic on $L_{\rho}^{p}(\mathbb{R})$. On the other hand,

$$
\begin{aligned}
& e^{-p s_{i} \lambda} \rho\left(s_{i}\right)=e^{-p \lambda s_{i}}, \\
& e^{p s_{i} \lambda} \rho\left(-s_{i}\right)=e^{\left(p \lambda-\frac{1}{2}\right) s_{i}} .
\end{aligned}
$$

Again, both sequences converge to 0 , thus $e^{-\lambda t} T(t)$ is hypercyclic.

\subsection{Example 4}

Let $X$ and $T(t)$ be chosen as in Example 3, and let $\lambda_{n}$ be any sequence in $\left(-\frac{1}{2 p}, \frac{1}{2 p}\right)$ converging to 0 . Then the semigroups $e^{\lambda_{n} t} T(t)$ are all hypercyclic, and obviously they converge to $T(t)$ in the operator norm, uniformly for $t$ in compact intervals. However, as shown above, $T(t)$ is not hypercylic.

\subsection{Example 5}

Before we construct our example, we prepare some technicalities about the Yosida approximation. For the beginning, let $T(t)$ any $C_{0}$-semigroup on some Banach space $X$ and let $A$ be its infinitesimal generator. We approximate $T(t)$ by its Yosida approximation $\exp \left(A_{\lambda} t\right)$ where

$$
A_{\lambda}=\lambda A(\lambda-A)^{-1}=\lambda^{2}(\lambda-A)^{-1}-\lambda \mathrm{id}
$$

which exists for sufficiently large $\lambda$.

Lemma 12. With the notation above, for all $t>0$ and all sufficiently large $\lambda$,

$$
\exp \left(A_{\lambda} t\right) x=e^{-\lambda t} x+\int_{0}^{\infty} g(\lambda, t, s) T(s) x d s
$$

where

$$
g(\lambda, t, s):=\sum_{j=0}^{\infty} \frac{\lambda^{2 j+2} t^{j+1} s^{j}}{(j+1) ! j !} e^{-\lambda(t+s)} .
$$


Moreover, the Laplace transform $h(\lambda, t, \sigma)$ of $g(\lambda, t, s)$ with respect to s satisfies

$$
h(\lambda, t, \sigma)=e^{-\lambda t}\left(e^{\frac{\lambda^{2} t}{\lambda+\sigma}}-1\right) .
$$

Proof.

$$
\begin{aligned}
\exp \left(A_{\lambda} t\right) x & =e^{-\lambda t} \sum_{j=0}^{\infty} \frac{\lambda^{2 j} t^{j}}{j !}(\lambda-A)^{-j} x \\
& =e^{-\lambda t}\left[x+\sum_{j=1}^{\infty} \frac{\lambda^{2 j} t^{j}}{j !}(\lambda-A)^{-j} x\right] \\
& =e^{-\lambda t}\left[x+\sum_{j=1}^{\infty} \frac{\lambda^{2 j} t^{j}}{j !} \int_{0}^{\infty} \frac{s^{j-1}}{(j-1) !} e^{-\lambda s} T(s) x d s\right] \\
& =e^{-\lambda t} x+\int_{0}^{\infty} \sum_{j=0}^{\infty} \frac{\lambda^{2 j+2} t^{j+1} s^{j}}{(j+1) ! j !} e^{-\lambda(t+s)} T(s) x d s \\
& =e^{-\lambda t} x+\int_{0}^{\infty} g(\lambda, t, s) T(s) x d s .
\end{aligned}
$$

For the Laplace transform of $g$ we obtain

$$
\begin{aligned}
h(\lambda, t, \sigma) & =\int_{0}^{\infty} e^{-\sigma s} g(\lambda, t, s) d s \\
& =\int_{0}^{\infty} \sum_{j=0}^{\infty} \frac{\lambda^{2 j+2} t^{j+1} s^{j}}{(j+1) ! j !} e^{-\lambda(t+s)} e^{-\sigma s} d s \\
& =\sum_{j=0}^{\infty} \frac{\lambda^{2 j+2} t^{j+1}}{(j+1) !(\lambda+\sigma)^{j+1}} e^{-\lambda t} \\
& =e^{-\lambda t}\left(e^{\frac{\lambda^{2} t}{\lambda+\sigma}}-1\right) .
\end{aligned}
$$

Lemma 13. Let $g(\lambda, t, s)$ be as in Lemma 12. By $\delta$ we denote Dirac's measure concentrated at 0. Then

$$
\tilde{g}(\lambda, t, \cdot):=g(\lambda, t, \cdot)+e^{-\lambda t} \delta
$$

is the density of some nonnegative random variable $Z_{\lambda, t}$. The expectation and variance of $Z_{\lambda, t}$ are given by

$$
E\left(Z_{\lambda, t}\right)=t, \quad \operatorname{Var}\left(Z_{\lambda, t}\right)=\frac{2 t}{\lambda}
$$


Proof. In fact, let

$$
\tilde{h}(\lambda, t, \sigma)=e^{-\lambda t}+h(\lambda, t, \sigma)=e^{-\frac{\lambda t \sigma}{\lambda+\sigma}}
$$

be the Laplace transform of $\tilde{g}(\lambda, t, \cdot)$. Then

$$
\int_{0}^{\infty} \tilde{g}(\lambda, t, s) d s=\tilde{h}(\lambda, t, 0)=1 .
$$

Moreover, for any power $k$, we can obtain the expectation

$$
E\left(Z_{\lambda, t}^{k}\right)=(-1)^{k} \frac{\partial}{\partial \sigma} \tilde{h}(\lambda, t, 0)
$$

which yields the expectation and the variance for $Z_{\lambda, t}$.

Lemma 14. With $g$ as in Lemma 12, there exists a continuous, nonincreasing function $w:[0, \infty) \rightarrow$ $[0, \infty)$, such that $\lim _{t \rightarrow \infty} w(t)=0$, and for all $t>0, \lambda>0$,

$$
\int_{0}^{\infty} g(\lambda, t, s)^{2} d s \leq \lambda w(\lambda t)
$$

Proof. By Plancherel's Theorem we have

$$
\begin{aligned}
& \int_{0}^{\infty} g(\lambda, t, s)^{2} d s \\
= & \frac{1}{\pi} \int_{0}^{\infty}|h(\lambda, t, i \sigma)|^{2} d \sigma \\
= & \frac{1}{\pi} \int_{0}^{\infty}\left|e^{-\lambda t}\left(e^{\frac{\lambda^{2} t}{\lambda+i \sigma}}-1\right)\right|^{2} d \sigma \\
& \left(\operatorname{setting} \tau:=\lambda t \text { and } \nu:=\frac{\sigma}{\lambda}\right) \\
= & \frac{\lambda}{\pi} \int_{0}^{\infty}\left|e^{-\tau}\left(e^{\frac{\tau}{1+i \nu}}-1\right)\right|^{2} d \nu \\
= & \frac{\lambda}{\pi} \int_{0}^{\infty}\left|e^{-\tau}\left(e^{\frac{\tau-i \nu \tau}{1+\nu^{2}}}-1\right)\right|^{2} d \nu \\
= & \frac{\lambda}{\pi} \int_{0}^{\infty} e^{-2 \tau}\left(e^{\frac{2 \tau}{1+\nu^{2}}}-2 e^{\frac{\tau}{1+\nu^{2}}} \cos \left(\frac{\nu \tau}{1+\tau^{2}}\right)+1\right) d \nu \\
= & \frac{\lambda}{\pi} \int_{0}^{\infty}\left[e^{-2 \tau}\left(e^{\frac{\tau}{1+\nu^{2}}}-1\right)^{2}+2 e^{-2 \tau} e^{\frac{\tau}{1+\nu^{2}}}\left(1-\cos \left(\frac{\nu \tau}{1+\nu^{2}}\right)\right)\right] d \nu \\
= & \lambda\left[w_{1}(\tau)+w_{2}(\tau)+w_{3}(\tau)\right]
\end{aligned}
$$


with

$$
\begin{aligned}
& w_{1}(\tau)=\frac{1}{\pi} \int_{0}^{1} e^{-2 \tau}\left(e^{\frac{\tau}{1+\nu^{2}}}-1\right)^{2} d \nu \\
& w_{2}(\tau)=\frac{1}{\pi} \int_{1}^{\infty} e^{-2 \tau}\left(e^{\frac{\tau}{1+\nu^{2}}}-1\right)^{2} d \nu \\
& w_{3}(\tau)=\frac{1}{\pi} \int_{0}^{\infty} 2 e^{-2 \tau} e^{\frac{\tau}{1+\nu^{2}}}\left(1-\cos \left(\frac{\nu \tau}{1+\nu^{2}}\right)\right) d \nu .
\end{aligned}
$$

It is easy to see that the integrands in all three integrals above converge to 0 for fixed $\nu$ as $\tau \rightarrow \infty$. In the first integral, the integrand can be bounded by the constant 4 . The integrand in the second integral is dominated by

$$
\begin{aligned}
& e^{-2 \tau}\left(e^{\frac{\tau}{1+\nu^{2}}}-1\right)^{2} \leq e^{-2 \tau}\left(e^{\frac{\tau}{1+\nu^{2}}} \frac{\tau}{1+\nu^{2}}\right)^{2}=e^{-\frac{2 \tau \nu^{2}}{1+\nu^{2}}}\left(\frac{\tau}{1+\nu^{2}}\right)^{2} \\
& \leq \tau^{2} e^{-\tau}\left(\frac{1}{1+\nu^{2}}\right)^{2} \leq 2\left(\frac{1}{1+\nu^{2}}\right)^{2}
\end{aligned}
$$

The third integrand is bounded by

$$
\begin{aligned}
& 2 e^{-2 \tau} e^{\frac{\tau}{1+\nu^{2}}}\left(1-\cos \left(\frac{\nu \tau}{1+\nu^{2}}\right)\right) \leq 2 e^{-\tau} e^{-\frac{\tau \nu^{2}}{1+\nu^{2}}}\left(2 \sin ^{2}\left(\frac{\nu \tau}{2\left(1+\nu^{2}\right)}\right)\right) \\
& \leq e^{-\tau}\left(\frac{\tau \nu}{1+\nu^{2}}\right)^{4} \leq \tau^{4} e^{-\tau}\left(\frac{\nu^{2}}{1+\nu^{2}}\right)^{2}\left(\frac{1}{1+\nu^{2}}\right)^{2} \leq M\left(\frac{1}{1+\nu^{2}}\right)^{2}
\end{aligned}
$$

with a suitable constant $M>0$. Therefore by the dominated convergence theorem, $w_{1}(\tau), w_{2}(\tau)$, and $w_{3}(\tau)$ converge to 0 as $\tau \rightarrow \infty$. Now put

$$
w(\tau)=\sup _{\sigma>\tau}\left[w_{1}(\sigma)+w_{2}(\sigma)+w_{3}(\sigma)\right]
$$

Lemma 15. With $g$ as in Lemma 12, the following inequalities hold for all $\lambda, t, u, \alpha>0, \beta \in(0, t)$ :

$$
\begin{aligned}
& e^{-\lambda t}+\int_{0}^{t-\beta} g(\lambda, t, s) d s+\int_{t+\beta}^{\infty} g(\lambda, t, s) d s \leq \frac{t}{\beta^{2} \lambda} \\
& \int_{s}^{s+\alpha} g(\lambda, t, s) d s \leq \sqrt{\alpha} \sqrt{\lambda w(\lambda t)} .
\end{aligned}
$$


Proof. Let $Z_{\lambda, t}$ be as in Lemma 13. Chebychev's inequality implies

$$
\begin{aligned}
\frac{2 t}{\lambda \beta^{2}} & \geq \mathbb{P}\left(\left|Z_{\lambda, t}-t\right| \geq \beta\right)=\int_{[0, t-\beta]} \tilde{g}(\lambda, t, s) d s+\int_{[t-\beta, \infty)} \tilde{g}(\lambda, t, s) d s \\
& =e^{\lambda t}+\int_{0}^{t-\beta} g(\lambda, t, s) d s+\int_{t-\beta}^{\infty} g(\lambda, t, s) d s .
\end{aligned}
$$

The second inequality of the lemma is a straightforward consequence of Lemma 14 and Hölder's inequality.

Lemma 16. Consider sequences $\left(a_{i}\right)$ and $\left(b_{i}\right)$ converging to infinity such that

$$
\begin{aligned}
& 0=b_{0}<a_{1}<b_{1}<a_{2}<\ldots \\
& a_{i} \geq b_{i-1}+2^{2 i} \\
& w\left(a_{i}\right) \leq 2^{-5 i} \\
& b_{i}=a_{i}+2^{i}
\end{aligned}
$$

and put

$$
q(t):= \begin{cases}2^{i} & \text { for } t \in\left[a_{i}, b_{i}\right] \\ 0 & \text { elsewhere }\end{cases}
$$

Then for each fixed $\lambda \geq 1$ we have

$$
\lim _{t \rightarrow \infty} \int_{0}^{\infty} g(\lambda, t, s) q(s+h) d s=0
$$

uniformly for $h$ in compact intervals.

Proof. To show this assertion, we fix some compact interval $[0, T]$ and $\lambda \geq 1$. Let $t>1$ be sufficiently large and choose $i$ such that

$$
a_{i} \leq t \leq t+T \leq b_{i+1} .
$$

Using Lemma 15 several times, we obtain for $0 \leq h \leq T$ :

$$
\begin{aligned}
& \int_{0}^{\infty} g(\lambda, t, s) q(s+h) d s \\
\leq & \int_{0}^{b_{i-1}-h} g(\lambda, t, s) 2^{i-1} d s+\int_{a_{i}-h}^{b_{i}-h} g(\lambda, t, s) 2^{i} d s \\
& +\int_{a_{i+1}-h}^{b_{i+1}-h} g(\lambda, t, s) 2^{i+1} d s+\sum_{k=2}^{\infty} \int_{a_{i+k}-h}^{b_{i+k}-h} 2^{i+k} g(\lambda, t, s) d s \\
\leq & 2^{i-1} \frac{2 t}{\left(t-b_{i-1}+h\right)^{2} \lambda}+2^{i} \sqrt{2^{i}} \sqrt{\lambda w(\lambda t)} \\
& +2^{i+1} \sqrt{2^{i+1}} \sqrt{\lambda w(\lambda t)}+\sum_{k=2}^{\infty} 2^{i+k} \frac{2 t}{\left(a_{i+k}-h-t\right)^{2} \lambda} .
\end{aligned}
$$


Note that $2^{2 i} b_{i-1} \leq a_{i} \leq t$, and therefore $\left(t-b_{i-1}+h\right) \geq t\left(1-2^{-2 i}\right) \geq \frac{t}{2}$. Thus

$$
\frac{2 t}{\left(t-b_{i-1}+h\right)^{2} \lambda} \leq \frac{8 t}{\lambda t^{2}} \leq \frac{8}{\lambda} 2^{-2 i}
$$

Similarly, we have for $k \geq 2$

$$
a_{i+k}-t-h \geq a_{i+k}-b_{i+k-1} \geq 2^{2(i+k)} b_{i+k-1} \geq 2^{2(i+k)} t .
$$

Hence

$$
\frac{2 t}{\lambda\left(a_{i+k}-h-t\right)^{2}} \leq \frac{2}{\lambda} 2^{-4(i+k)}
$$

Thus

$$
\begin{aligned}
& \int_{0}^{\infty} q(s+h) g(\lambda, t, s) d s \\
\leq & 2^{i-1} \frac{8}{\lambda} 2^{-2 i}+2^{\frac{3 i}{2}} \sqrt{\lambda} 2^{-\frac{5 i}{2}} \\
& +2^{\frac{3(i-1)}{2}} \sqrt{\lambda} 2^{-\frac{5(i+1)}{2}}+\sum_{k=2}^{\infty} 2^{i+k} \frac{2}{\lambda} 2^{-4(i+k)} \\
= & \frac{1}{\lambda} 2^{-i+2}+\sqrt{\lambda}\left(2^{-i}+2^{-i-1}\right)+\frac{2}{\lambda} 8^{-i-1} \frac{1}{1-\frac{1}{8}} .
\end{aligned}
$$

As $t \rightarrow \infty$, we have also $i \rightarrow \infty$, hence and the right hand side of the last estimate converges to 0 as claimed.

Construction of Example 5:

Let $0=a_{1} \leq b_{1}<a_{2}<b_{2} \cdots$ be as in Lemma 16. We construct an admissible weight function $\rho:[0, \infty) \rightarrow[0, \infty)$ with the following properties

$$
\begin{aligned}
& \rho(t)=1 \quad \text { for } t \in\left[b_{i}, a_{i+1}\right] \\
& 2^{-i} \leq \rho(t) \leq 1 \quad \text { on }\left[a_{i}, b_{i}\right] \\
& \rho(t) \leq e^{h} \rho(t+h) \quad \text { for all } t \geq 0, h \geq 0 \\
& \lim \inf _{t \rightarrow \infty} \rho(t)=0 .
\end{aligned}
$$

This can be easily achieved since the gaps $b_{i}-a_{i}$ tend to infinity as $i \rightarrow \infty$. Notice that with the function $q$ in Lemma 16 we have for all $t \geq 0, h \geq 0$

$$
\frac{\rho(h)}{\rho(t)} \leq \frac{1}{\rho(t)} \leq \max (1, q(t)) \leq 1+q(t)
$$


Now fix any (nonzero) $\phi \in \mathcal{C}_{0, \rho}([0, \infty)), \lambda \geq 1$, and $T \geq 0$. For all $h \in[0, T]$ we have

$$
\begin{aligned}
& \rho(h)\left|\left[\exp \left(A_{\lambda} t\right) \phi\right](h)\right| \\
= & \rho(h)\left|e^{-\lambda t} \phi(h)+\int_{0}^{\infty} g(\lambda, t, s) \phi(s+h) d s\right| \\
\leq & e^{-\lambda t} \rho(h)|\phi(h)|+\int_{0}^{\infty} g(\lambda, t, s) \frac{\rho(h)}{\rho(s+h)} \rho(s+h)|\phi(s+h)| d s \\
\leq & e^{-\lambda t}\|\phi\|+\int_{0}^{\infty} g(\lambda, t, s)(1+q(s+h))\|\phi\| d s \\
= & {\left[e^{-\lambda t}+\int_{0}^{\infty} g(\lambda, t, s) d s+\int_{0}^{\infty} g(\lambda, t, s) q(s+h) d s\right]\|\phi\| } \\
= & {\left[1+\int_{0}^{\infty} g(\lambda, t, s) q(s+h) d s\right]\|\phi\| . }
\end{aligned}
$$

The last equality holds since $e^{-\lambda t} \delta+g(\lambda, t, \cdot)$ is a probability density function (Lemma 13). Now we infer from Lemma 16 that

$$
\lim \sup _{t \rightarrow \infty} \sup _{h \in[0, T]} \rho(h)\left|\left[\exp \left(A_{\lambda} t\right) \phi\right](h)\right| \leq\|\phi\|
$$

Therefore, for any vector $\psi \in \mathcal{C}_{0, \rho}([0, \infty))$ with

$$
\sup _{h \in[0, t]} \rho(h)|\psi(h)| \geq 2\|\phi\|
$$

we have

$$
\lim \inf _{t \rightarrow \infty}\left\|\left[\exp \left(A_{\lambda} t\right) \phi\right]-\psi\right\| \geq\|\phi\| .
$$

Consequently, the trajectory of $\phi$ is not dense, and the semigroups $\exp \left(A_{\lambda} t\right)$ are not hypercyclic.

\subsection{Example 6}

Our last example will use another standard class of hypercyclic semigroups: Let $\rho_{k}$ be a sequence in $\mathbb{R}$ with the following properties

$$
\rho(k)>0, \quad \sup _{k \in \mathbb{N}} \frac{\rho_{k}}{\rho_{k+1}}<\infty .
$$

Let $X:=l_{\rho}^{p}$ be the set of all sequences $\left(x_{n}\right)$ such that

$$
\left\|\left(x_{k}\right)\right\|^{p}:=\sum_{k=0}^{\infty} \rho_{k}\left|x_{k}\right|^{p}
$$


is finite. With the positive cone

$$
X^{+}=\left\{\left(x_{n}\right) \in l_{\rho}^{p} \mid x_{n} \geq 0 \text { for all } n \in \mathbb{N}\right\},
$$

the space $l_{\rho}^{p}$ is a Banach lattice.

We define the left shift $A$ in $l_{\rho}^{p}$ by

$$
A x=y \quad \text { with } \quad y_{k}=x_{k+1} .
$$

Then $A$ is a bounded linear operator, in particular it generates an analytic semigroup $T(t)$. It is known [5] that $T(t)$ is hypercyclic. Since $A\left(X^{+}\right) \subset X^{+}$, we infer that $T(t)$ is nonnegative in the sense that $T(t)\left(X^{+}\right) \subset X^{+}$. Moreover, for $x \in X^{+}$we have

$$
\frac{d}{d t} T(t) x=A T(t) x \in X^{+}
$$

so that $T(t) x \geq T(s) x$ if $t \geq s$. Since for $0 \neq x \in X^{+}$the set $\left\{y \in l_{\rho}^{p} \mid y \geq x\right\}$ is a proper closed subset of $X^{+}$, the trajectory $\{T(t) x \mid t \geq 0\}$ is not dense in $X^{+}$.

\section{References}

[1] T. Bermudez, A. Bonilla, J.A. Conejero, A. Peris. Hypercyclicity, topologically mixing and chaotic semigroups on Banach spaces. Studia Math., 170 (2005), 57-75.

[2] J. Banasiak, M. Lachowicz. Chaotic linear dynamics with applications. SOTA 2 (2001).

[3] J. Banasiak, M. Lachowicz, M. Moszynski. When topology meets medicine. Applied Math. Letters, 16 (2003) 303-308.

[4] V.A. Conejero, V. Mller, A. Peris. Hypercyclic behaviour of operators in a hypercyclic $C_{o}$ semigroup. J. Functional Analysis, 244 (2007), 342-348.

[5] Desch W., W. Schappacher, G.F. Webb. Hypercyclic and chaotic semigroups of linear operators. Ergodic Theory and Dynamical Systems, 17 (1997), 793-819.

[6] H. Emamirad. Hypercyclicity in the scattering theory for linear transport equation. Trans. Amer. math. Soc., 350 (1998), 3707-3716.

[7] K.J. Engel, R. Nagel. One-parameter semigroups for linear evolution equations. Springer, 1999.

[8] K.G. Grosse-Erdmann. Universal families and hypercyclic operators. Bull. Amer. math. Soc., 36 (1999), 345-385.

[9] K. Howard. A size structured model of cell dwarfism. Discrete and Continuous Dynamical Systems, Ser. B., 1 (2002), 471-484 